\title{
Primer hallazgo de Pseudorasbora parva (Temminck \& Schlegel, 1846) (Cyprinidae) en la Cuenca Sur de España
}

\author{
Elías D. Dana ${ }^{1, *}$, Juan García de Lomas ${ }^{1}$, David M. García-Ocaña ${ }^{2}$, Vanesa Gámez² ${ }^{2}$ Jose M. \\ Ortíz $^{3}$, Francisco J. Galindo ${ }^{2}$, Silvia Rubio ${ }^{2}$ y Guillermo Ceballos ${ }^{4}$ \\ ${ }^{1}$ Agencia de Medio Ambiente y Agua de Andalucía, Johan Gutenberg 1, 41092, Sevilla (España). \\ 2 Agencia de Medio Ambiente y Agua de Andalucía, C/Joaquina Eguaras 10, Bajos del Edificio Victoria, 18013, \\ Granada (España). \\ ${ }^{3}$ Oficina Administrativa Agentes de Medio Ambiente. Plaza de Andalucía 22, 11350-Castellar de la Frontera, \\ Cádiz (España). \\ ${ }^{4}$ Consejería de Medio Ambiente y Ordenación del Territorio, Av. Manuel Siurot 50, 41071, Sevilla (España). \\ *Autor responsable de la correspondencia: edana@agenciamedioambienteyagua.es; edana@ual.es
}

Recibido: $23 / 07 / 2014$

Aceptado: 29/01/2015

\section{RESUMEN}

Primer hallazgo de Pseudorasbora parva (Temminck \& Schlegel, 1846) (Cyprinidae) en la Cuenca Sur de España

Como parte de los trabajos de evaluación de poblaciones nativas y detección temprana de especies invasoras que viene realizando la Consejería de Medio Ambiente y Ordenación del Territorio (Junta de Andalucía), en la Cuenca del Sur (Ríos Hozgarganta y Guadiaro), se ha detectado Pseudorasbora parva Temminck \& Schlegel (Cyprinidae, subfamilia Gobioninae), un pez invasor procedente de Asia. En esta nota se discuten las posibles vías de introducción, los impactos asociados y su posible repercusión sobre especies nativas de la región, y se plantean las líneas generales para futuras medidas de gestión. Resulta especialmente preocupante tanto la esperable dispersión hacia otros cauces y cuencas como la potencial transmisión de dos parásitos, también alóctonos (Sphaerotecum destruens y Anguillicola crassus) a poblaciones de especies autóctonas. Aunque se desconoce el origen de esta introducción en la región, el historial de uso de la especie en Europa y España, hace pensar que haya podido ser introducido inicialmente como pez pasto para especies predadoras pescables como Micropterus salmoides, dispersándose después entre masas de aguas conectadas. La erradicación o el control a través de la gestión directa de la población no parecen factibles. Por ello proponemos que las medidas de gestión futuras contemplen, entre otros aspectos, el seguimiento del estado de las poblaciones de especies autóctonas de mayor valor para la conservación y labores de difusión entre colectivos y administraciones implicadas acerca del potencial impacto que la especie puede provocar en la ictiofauna del Sur de España.

Palabras clave: Invasiones biológicas, alóctonas, invasoras, parásitos ictícolas.

\begin{abstract}
First record of Pseudorasbora parva (Temminck \& Schlegel, 1846) (Cyprinidae) in the Cuenca Sur of Spain

Pseudorasbora parva Temminck \& Schlegel (Cyprinidae, subfamily Gobioninae), an invasive Asian fish, has been found in the Cuenca Sur (Hozgarganta and Guadiaro rivers) for the first time thanks to the field works for the assessment of native aquatic fauna and for the early detection of invasive species. These works are being conducting by the Environmental Council (Regional Government of Andalusia). In this work, potential introduction routes and associated biological impacts on native fish species are evaluated, and suggestions for the design of future management strategies are presented. It is expected that $\mathrm{P}$. parva will disperse through other neighbor watercourses and basins. There might be also a risk of transmission of two alien parasites (Sphaerotecum destruens y Anguillicola crassus) to the populations of native fish species. The introduction route for P. parva in the Cuenca Sur is unknown. However, it is likely that the species was first introduced as forage fish to promote angling species such as Micropterus salmoides, already present in the rivers, and thus invade connected water bodies. Eradication or control through direct population management seems unfeasible. Therefore, future management measures focused on monitoring and management of native fish populations are proposed as well as the development of awareness campaigns involving all the stakeholders.
\end{abstract}

Key words: Biological invasions, alien species, invasive species, fish parasites. 


\section{INTRODUCCIÓN}

Pseudorasbora parva (Temminck \& Schlegel, 1846) (Cyprinidae, subfamily Gobioninae) es un pez nativo del Este de Asia (Japón, Korea, Norte y Centro de China y Sureste de la antigua URSS) que ha sido introducido en numerosas regiones de Europa, Asia Menor y África (Gozlan et al., 2002). En Europa se detectó en la década de 1960 en Rumanía, donde pudo ser introducida de manera accidental acompañando alevines de carpa (Banarescu, 1964). Posteriormente ha ido avanzando progresivamente por numerosos países europeos, en unos casos por traslocaciones y nuevas introducciones intencionadas como pez pasto, y en otros, por la dispersión de las poblaciones a través de masas de agua conectadas (Gozlan et al., 2010).
Esta especie invasora provoca impactos como consumidora de algas, crustáceos planctónicos y alevines de otras especies, lo que genera cambios importantes en las dinámicas tróficas (Gozlan et al., 2010; Jackson \& Britton, 2013). Además, $P$. parva actúa como vector de, al menos, dos especies parásitas generalistas: Sphaerothecum destruens y Anguillicola crassus. Sphaerothecum destruens es un parásito intracelular eucariota con una fase de vida libre como zoospora infestante (Mendoza et al., 2002; Arkush et al., 2003) que produce inflamación, serositis y muerte celular en los órganos infectados, provocando mortandad crónica y sostenida de ejemplares de las poblaciones afectadas (Andreou et al., 2012). Esta patología afecta, entre otras especies, a la carpa común (Cyprinus carpio), la brema (Abramis brama), el rutilo (Rutilus rutilus) y a los gé-

Tabla 1. Citas publicadas de P. parva en España. Las Coordenadas geográficas se refieren al Datum: WGS84; Elipsoide: WGS84. Las Proyectadas se ofrecen en UTM (Universal Transverse Mercator), corregidas para el Huso 30S (Datum: ED-1950; Elipsoide: International 1924; zona 30). Published records for P. parva in Spain. Geographic Coordinates are referred to Datum: WGS84; Ellipsoid: WGS84. Projected coordinates are in UTM (Universal Transverse Mercator) System, corrected for the Huse $30 S$ (Datum: ED-1950; Ellipsoid: International 1924; zone 30).

\begin{tabular}{|c|c|c|c|c|c|}
\hline $\begin{array}{l}\text { Coordenadas } \\
\text { Geográficas }\end{array}$ & $\begin{array}{c}\text { Coordenadas } \\
\text { proyectadas (UTM) }\end{array}$ & Año & Río/Localidad & Cuenca & Referencia \\
\hline $\begin{array}{l}\mathrm{N} 40^{\circ} 33^{\prime} \text { to } 40^{\circ} 48^{\prime} ; \\
\mathrm{E} 0^{\circ} 39^{\prime} \text { to } 0^{\circ} 53^{\prime}\end{array}$ & $\begin{array}{c}810195 ; 4496789 \text { to } \\
827727 ; 4524026\end{array}$ & 2001 & Ebro & Ebro & $\begin{array}{c}\text { Caiola \& De Sostoa } \\
\text { (2002) }\end{array}$ \\
\hline $\begin{array}{l}\mathrm{N} 42^{\circ} 1^{\prime} 41^{\prime \prime} ; \\
\mathrm{E} 3^{\circ} 5^{\prime} 46^{\prime \prime}\end{array}$ & $508057 ; 4653104$ & 2007 & Daró & $\begin{array}{c}\text { Cuencas Internas de } \\
\text { Cataluña }\end{array}$ & $\begin{array}{c}\text { Pou-Rovira et al. } \\
\text { (2007); Clavero, com. } \\
\text { pers. }\end{array}$ \\
\hline $\begin{array}{l}\mathrm{N} 42^{\circ} 5^{\prime} 43.8^{\prime \prime} \\
\mathrm{E} 3^{\circ} 6^{\prime} 55.1^{\prime \prime}\end{array}$ & $509633 ; 4659357$ & 2007 & Rec de Cinyana & $\begin{array}{c}\text { Cuencas Internas de } \\
\text { Cataluña }\end{array}$ & $\begin{array}{c}\text { Pou-Rovira et al. } \\
\text { (2007); Clavero, com. } \\
\text { pers. }\end{array}$ \\
\hline $\begin{array}{l}\mathrm{N} 42^{\circ} 5^{\prime} 11.1^{\prime \prime} \\
\mathrm{E} 3^{\circ} 7^{\prime} 46.2^{\prime \prime}\end{array}$ & $510807 ; 4659582$ & 2007 & Rec de Cinyana & $\begin{array}{l}\text { Cuencas Internas de } \\
\text { Cataluña }\end{array}$ & $\begin{array}{c}\text { Pou-Rovira et al. } \\
\text { (2007); Clavero, com. } \\
\text { pers. }\end{array}$ \\
\hline $\begin{array}{l}\mathrm{N} 42^{\circ} 6^{\prime} 14.3^{\prime \prime} \\
\mathrm{E} 3^{\circ} 6^{\prime} 14.6^{\prime \prime}\end{array}$ & $508700 ; 4661528$ & 2007 & Riera de les Llones & $\begin{array}{c}\text { Cuencas Internas de } \\
\text { Cataluña }\end{array}$ & $\begin{array}{c}\text { Pou-Rovira et al } \text {. } \\
\text { (2007); Clavero, com } \text {. } \\
\text { pers. }\end{array}$ \\
\hline $\begin{array}{l}\mathrm{N} 42^{\circ} 7^{\prime} 20.1^{\prime \prime} \\
\mathrm{E} 3^{\circ} 6^{\prime} 56.1^{\prime \prime}\end{array}$ & $509651 ; 4663559$ & 2007 & Rec del Molí de l'Escala & $\begin{array}{l}\text { Cuencas Internas de } \\
\text { Cataluña }\end{array}$ & $\begin{array}{c}\text { Pou-Rovira et al. } \\
\text { (2007); Clavero, com. } \\
\text { pers. }\end{array}$ \\
\hline $\begin{array}{l}\mathrm{N} 42^{\circ} 0^{\prime} 53.5^{\prime \prime} \\
\mathrm{E} 3^{\circ} 6^{\prime} 49.2^{\prime \prime}\end{array}$ & $509508 ; 4651635$ & 2007 & $\begin{array}{c}\text { Daró Vell-Rec del Molí } \\
\text { de Pals }\end{array}$ & $\begin{array}{l}\text { Cuencas Internas de } \\
\text { Cataluña }\end{array}$ & $\begin{array}{c}\text { Pou-Rovira et al. } \\
\text { (2007); Clavero, com. } \\
\text { pers. }\end{array}$ \\
\hline $\begin{array}{l}\mathrm{N} 42^{\circ} 5^{\prime} 34.6^{\prime \prime} \\
\mathrm{E} 3^{\circ} 6^{\prime} 31.3^{\prime \prime}\end{array}$ & $509086 ; 4660305$ & 2007 & Rec del Molí de l'Escala & $\begin{array}{l}\text { Cuencas Internas de } \\
\text { Cataluña }\end{array}$ & $\begin{array}{c}\text { Pou-Rovira et al. } \\
\text { (2007); Clavero, com. } \\
\text { pers. }\end{array}$ \\
\hline $\begin{array}{l}\mathrm{N} 40^{\circ} 43^{\prime} 12^{\prime \prime} \\
\mathrm{E} 0^{\circ} 51^{\prime} 47^{\prime \prime}\end{array}$ & $826408 ; 4515066$ & 2010 & Ebro & Ebro Delta & Simon et al. (2011) \\
\hline $\begin{array}{l}\mathrm{N} 39^{\circ} 19^{\prime} 21^{\prime \prime} \\
\mathrm{W} 5^{\circ} 46^{\prime} 36^{\prime \prime}\end{array}$ & $260746 ; 4356449$ & 2010 & Alcollarín & Guadiana & Aparicio et al. (2012) \\
\hline
\end{tabular}


neros Oncorhynchus, Salmo y Salvelinus (Harrell et al., 1986; Hedrick et al., 1989; Arkush et al., 1998; Gozlan et al., 2006, 2009; Andreou et al., 2012). La prevalencia en poblaciones silvestres de especies de peces autóctonos europeas es prácticamente desconocida, aunque recientemente se ha estimado una prevalencia en poblaciones holandesas de Pseudorasbora parva entre el $67 \%$ y el 74 \% (Spikmans et al., 2013). Anguillicola crassus es un nematodo asiático que, en su región de origen, parasita a Anguilla japonica. Este parásito ya está presente en ríos ibéricos afectando a A. anguilla (Gallastegi et al., 2002, Maillo et al., 2005), que resulta ser más susceptible que A. japonica (Knopf \& Mahnke, 2004) y se suma a otras especies de parásitos introducidas con la llegada de especies de peces invasores (García-Berthou et al., 2007). A. crassus provoca debilitamiento y reducción de la tolerancia al estrés ambiental en los peces afectados, llevándoles incluso a la muerte. Al afectarse a la vejiga natatoria, impide la normal natación de los peces y la migración (EIFAC \& ICES, 2008; Denny et al., 2013). Por ello, la introducción de $P$. parva puede conllevar la extinción local de especies con las que coexiste.

En España, la venta y tenencia de $P$. par$v a$ se encuentra prohibida por el Real Decreto 630/2013 (BOE, 2013). Su introducción ha sido reciente y los hallazgos, casuales, a menudo coincidiendo con muestreos de especies nativas (Tabla 1). La primera cita data de 2001 para el Delta del Ebro (NE España) (Caiola \& De Sostoa, 2002; Simon et al., 2011), quizá escapada de instalaciones de cría de ciprínidos para acuariofilia próximas a la zona de estudio. En 2005 se encontró en los ríos Ter y Daró (Cuencas Internas de Cataluña) (Pou-Rovira et al., 2007; Clavero, com. pers.). Posteriormente, en 2010, se encontró en la Cuenca del Guadiana (Río Alcollarín, tributario del Guadiana, Aparicio et al., 2012).

En este artículo se reporta la primera cita de Pseudorasbora parva en la Cuenca Sur de España, se discuten las posibles vías de introducción, se evalúan los posibles impactos esperables sobre las especies nativas de la región y se sugieren, brevemente, algunas líneas generales de gestión de este nuevo caso de invasión.

\section{MATERIAL Y MÉTODOS}

\section{Área de estudio}

Los ríos Hozgarganta y Guadiaro se encuentran en el Sur de la Península Ibérica y forman parte de la Subcuenca del Guadiaro, de $148.42 \mathrm{~km}^{2}$, incluida en la Cuenca del Sur. Constituye el área de drenaje de los relieves orientales del Aljibe. El Río Hozgarganta se une al Río Guadiaro, a unos $7 \mathrm{~km}$ de su desembocadura. El clima es de tipo mediterráneo, con inviernos suaves y húmedos y veranos secos y calurosos. En la localidad más próxima con estación meteorológica (Jimena de la Frontera) la temperatura y precipitación media es de $17.9^{\circ} \mathrm{C}$ y $739 \mathrm{~mm} / \mathrm{año}$. Este trabajo surge a raíz de unos muestreos caracterizadores de las comunidades de peces autóctonos en el Río Hozgarganta (Fig. 1) y como parte de los trabajos de prospección y alerta temprana de especies invasoras que viene realizando la Consejería de Medio Ambiente y Ordenación del Territorio (Junta de Andalucía).

El Río Hozgarganta forma parte del Parque Natural de Los Alcornocales y de la Red Natura 2000. Está catalogado, junto a sus afluentes y al Río Guadiaro como Lugar de Interés Comunitario (LIC) (ES6120031) con hábitats de interés comunitario incluidos en la Directiva 92/43/CE: 3150 lagos eutróficos con Magnopotamion o Hydrocharition, 91B0: Bosques termófilos de Fraxinus angustifolia, 92A0: Bosques de Galería de Salix alba y Populus alba, 92D0: Galerías y matorrales ribereños termomediterráneos (NerioTamaricetea y Securinegion tinctoriae), 92B0: Bosques de galería de ríos mediterráneos de caudal intermitente, con Rhododendron ponticum, Salix spp. así como otros taxones y comunidades biológicas de interés (European Environment Agency, 2009; Pérez-Latorre et al., 1999; JuradoDoña, 2002; Prenda et al., 2003; Ruiz-García et al., 2006; Tabla 2). Algunos de los taxones presentes están legalmente protegidos por la legislación (Directiva 92/43/CEE, Anexo II, DO 1992; Real Decreto 139/2011, BOE, 2011; Decreto 23/2012, BOJA, 2012). Los tramos medios y bajos están antropizados, con diversas especies alóctonas como Eucalyptus camaldulensis, 
aunque conservan restos de tarayal (Tamarix africana) y de fresneda termófila dominada por Fraxinus angustifolia.

\section{Muestreos de campo}

Los muestreos se enmarcan en los trabajos de detección de especies amenazadas y de especies invasoras que viene realizando la Consejería de Medio Ambiente y Ordenación del Territorio. Se llevaron a cabo entre septiembre de 2013 y mayo de 2014, mediante pasadas únicas de pesca eléctrica (200-250 V, 2-2.5 A, Pulsed DC) y artes pasivas en los puntos en los que resultaba muy complicado emplear la pesca eléctrica. Se emplearon entre 20 y 50 nasas cangrejeras $(5 \mathrm{~mm}$ de luz de malla) en cada punto ( 24 horas de permanencia). En conjunto, se muestrearon 21 puntos de los ríos Hozgarganta y Guadiaro (Fig. 1).

\section{RESULTADOS Y DISCUSIÓN}

En tres de los 21 puntos de muestreo apareció P. parva (Ver material suplementario, Fig. S1. Disponible en www.limnetica.net/internet): 2 de octubre de 2013 (N36 19'54"; W5 $22^{\prime} 02^{\prime \prime}$; $\left.L_{T}=50-110 \mathrm{~mm}, n=27\right)$ y 10 de abril de 2014 (N36 $25^{\prime} 44^{\prime \prime} ; \mathrm{W}^{\circ} 27^{\prime} 00^{\prime \prime} ; L_{T}=79 \mathrm{~mm}, n=1$ ) en el Río Hozgarganta, y el 23 de mayo (N36 19'10"; W5 $18^{\prime} 46^{\prime \prime} ; L_{T}=72 \mathrm{~mm}, n=1$ ) en el Río Guadiaro, a $26.4 \mathrm{~km}$ del primer punto (Fig. 1).

Pseudorasbora parva está experimentando una rápida expansión en España. Desde 2001 se viene produciendo un paulatino avance de manera discontinua con grandes saltos territoriales. Las citas aportadas en este trabajo constituyen las primeras dadas para la Cuenca Sur de España y las más meridionales conocidas

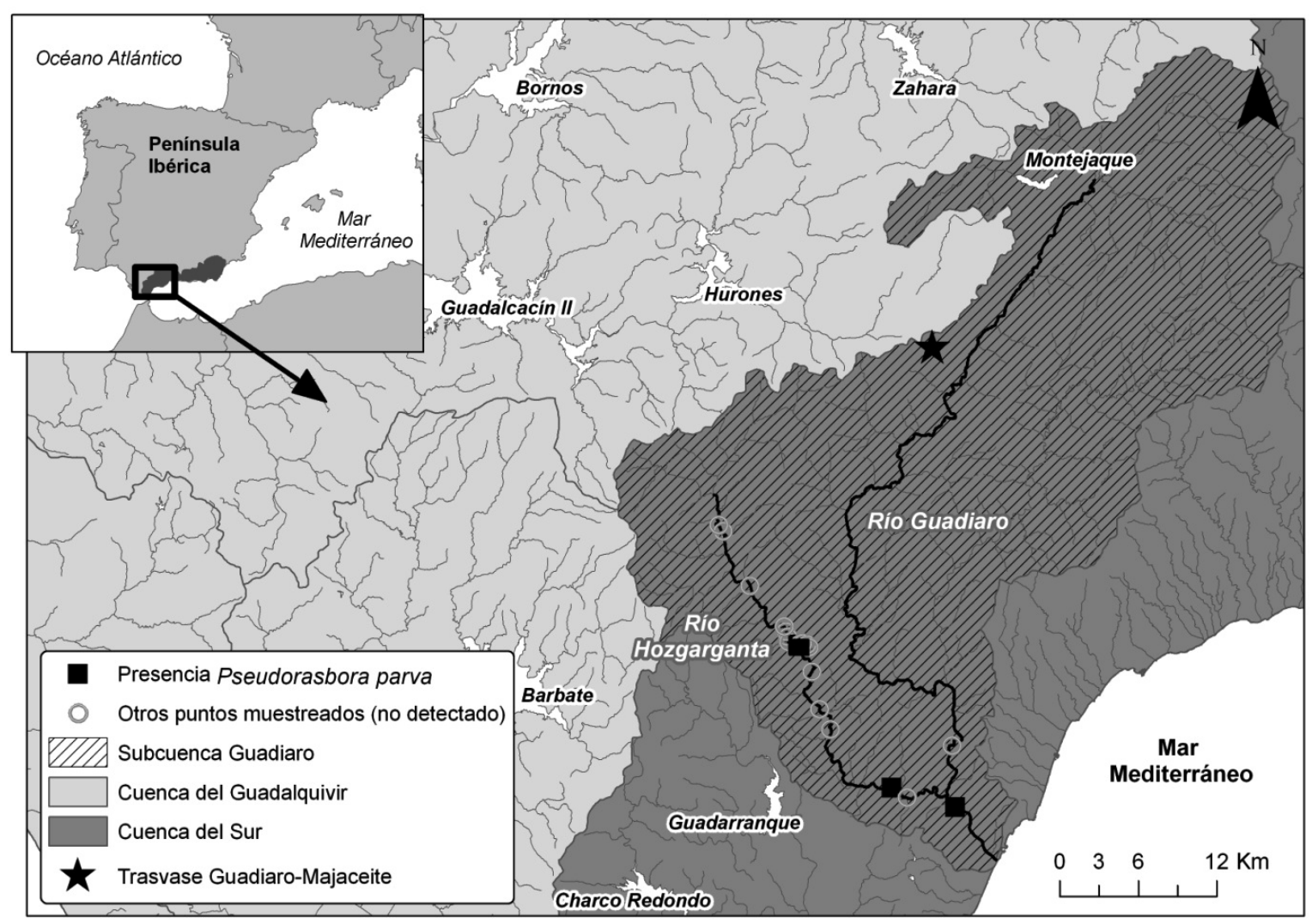

Figura 1. Distribución conocida de Pseudorasbora parva en Andalucía. Existen numerosos embalses en las cercanías del área de estudio. Known distribution of Pseudorasbora parva in Andalusia. Several reservoirs exist near the studied area. 
para esta especie en Europa. En la Cuenca Sur, la presencia de la especie se ha confirmado en tres puntos distantes, con especímenes capturados en años diferentes y correspondientes a clases de edad adulta reproductora (longitud furcal > $24 \mathrm{~mm}$; Pollux \& Korosi, 2006). En esta especie, por su carácter prolífico y por el rápido crecimiento de los juveniles, unos pocos ejemplares (1-5 parejas) bastan para producir importantes poblaciones en poco tiempo (Britton

Tabla 2. Principales especies de interés ligadas al medio acuático en los ríos Hozgarganta y Guadiaro. Fuentes: ${ }^{1}$ VVAA (2001), ${ }^{2}$ Doadrio et al. (2011) - en los casos en los que la clasificación de amenaza difería entre las fuentes se ha indicado la clasificación otorgada por cada fuente con el subíndice correspondiente $-{ }^{3}$ Barea-Azcón et al. (2008), ${ }^{4}$ Verdú \& Galante (2009), ${ }^{5}$ DO (1992), ${ }^{6}$ BOJA (2012), ${ }^{7}$ BOE (2011), ${ }^{8}$ BOE (2003). Para los peces, la nomenclatura sigue a Leunda et al. (2009) y para las especies no recogidas allí, a VVAA (2001) y a Barea-Azcón et al. (2008). ISCA = Incluido pero sin categoría de amenaza. Main taxa of interest linked to aquatic ecosystems in rivers Hozgarganta and Guadiaro. Sources: ${ }^{1}$ VVAA (2001), ${ }^{2}$ Doadrio et al. (2011) -in cases with lack of consensus about threaten category, the classifications provided by the two sources are shown-, ${ }^{3}$ Barea-Azcón et al. (2008), ${ }^{4}$ Verdú \& Galante (2009), ${ }^{5}$ DO (1992), ${ }^{6}$ BOJA (2012), ${ }^{7}$ BOE (2011), ${ }^{8}$ BOE (2003). For fish, nomenclature follows Leunda et al. (2009); for the species not listed there, nomenclature follows VVAA (2001) and Barea-Azcón et al. (2008). ISCA = the species is included, but its threaten category was not provided.

\begin{tabular}{|c|c|c|c|c|c|c|c|}
\hline \multirow[b]{2}{*}{ Especie } & \multicolumn{2}{|c|}{$\begin{array}{c}\text { CATEGORÍA DE } \\
\text { AMENAZA }\end{array}$} & \multirow[b]{2}{*}{ Europa $^{5}$} & \multicolumn{2}{|l|}{$\begin{array}{c}\text { PROTECCIÓN } \\
\text { LEGAL }\end{array}$} & \multirow[t]{2}{*}{$\begin{array}{l}\text { ¿AMENAZADA Y } \\
\text { NO PROTEGIDA? }\end{array}$} & \multirow[t]{2}{*}{$\begin{array}{c}\text { Especies } \\
\text { Pescables }^{8}\end{array}$} \\
\hline & Andalucía & España & & Andalucía ${ }^{6}$ & España $^{7}$ & & \\
\hline \multicolumn{8}{|l|}{ Peces } \\
\hline Anguilla anguilla & $\mathrm{LR}, \mathrm{nt}^{1}$ & $\mathrm{VU}^{1,2}$ & - & - & - & Sí & $\mathrm{X}$ \\
\hline Atherina boyeri & - & $\mathrm{VU}^{2}$ & - & - & - & Sí & \\
\hline $\begin{array}{l}\text { Barbus sclateri (Syn., } \\
\text { Luciobarbus sclateri) }\end{array}$ & $\mathrm{LR}, \mathrm{nt}^{1}$ & $\mathrm{LR}, \mathrm{nt}^{1,2}$ & - & - & - & Sí & $\mathrm{X}$ \\
\hline $\begin{array}{l}\text { Pseudochondrostoma } \\
\text { willkommii (Syn., } \\
\text { Chondrostoma } \\
\text { willkommii) }\end{array}$ & $\mathrm{VU}^{1}$ & $\mathrm{VU}^{1,2}$ & $\mathrm{X}$ & - & - & No & $\mathrm{X}$ \\
\hline Petromyzon marinus & $\mathrm{EN}^{1}$ & $\mathrm{VU}^{1,2}$ & $\mathrm{X}$ & En Peligro de Extinción & En peligro & No & \\
\hline Salaria fluviatilis & $\mathrm{CR}^{1}$ & $\mathrm{EN}^{1} / \mathrm{VU}^{2}$ & - & Vulnerable & VU & No & \\
\hline Squalius malacitanus & - & $\mathrm{CR}^{2}$ & - & - & - & Sí & \\
\hline $\begin{array}{l}\text { Squalius pyrenaicus } \\
\text { (Syn. Leuciscus } \\
\text { pyrenaicus) }\end{array}$ & $\mathrm{VU}^{1}$ & $\mathrm{VU}^{1,2}$ & - & - & - & Sí & $\mathrm{X}$ \\
\hline \multicolumn{8}{|l|}{ Macro-invertebrados } \\
\hline Oxygastra curtisii & $\mathrm{EN}^{3}$ & $\mathrm{EN}^{4}$ & $\mathrm{X}$ & Vulnerable & VU & No & \\
\hline Gomphus graslinii & $\mathrm{EN}^{3}$ & $\mathrm{EN}^{4}$ & $\mathrm{X}$ & Vulnerable & ISCA & No & \\
\hline Gomphus simillimus & $\mathrm{VU}^{3}$ & $\mathrm{VU}^{4}$ & - & Vulnerable & - & Sí & \\
\hline Macromia splendens & $\mathrm{CR}^{3}$ & $\mathrm{CR}^{4}$ & $\mathrm{X}$ & En Peligro de Extinción & En peligro & No & \\
\hline Anodonta anatina & $\mathrm{NT}^{3}$ & $\mathrm{LC}^{4}$ & - & - & - & Sí & \\
\hline Potomida littoralis & $\mathrm{VU}^{3}$ & $\mathrm{VU}^{4}$ & - & Listado Protección especial & - & No & \\
\hline Unio pictorum & $\mathrm{VU}^{3}$ & $\mathrm{NT}^{4}$ & - & - & - & Sí & \\
\hline Oestophora granesae & $\mathrm{EN}^{3}$ & $\mathrm{VU}^{4}$ & - & Listado Protección especial & - & No & \\
\hline Reptiles & & & & - & & & \\
\hline Emys orbicularis & $\mathrm{VU}^{1}$ & $\mathrm{VU}^{1,2}$ & $\mathrm{X}$ & - & ISCA & No & \\
\hline Mauremys leprosa & - & - & $\mathrm{X}$ & - & ISCA & No & \\
\hline
\end{tabular}


\& Gozlan, 2013). Por ello, cabe pensar que ya la reproducción y el reclutamiento efectivo se estén dando, especialmente si tenemos en cuenta que los ejemplares capturados son adultos y que se han capturado en dos ríos distintos. No obstante, para confirmar la dinámica poblacional y el área de extensión total en la cuenca serán necesarios futuros trabajos complementarios.

Se desconoce el origen y motivo de esta introducción aunque dado que no existen instalaciones de piscicultura, el escape puede descartarse. No obstante, existen al menos tres posibles circunstancias que podrían explicar su presencia. Una primera posibilidad sería la introducción intencionada debido al uso de esta especie como pez pasto, ya que en el tramo bajo del Río Guadiaro es habitual la pesca recreativa (p. ej.: sobre black bass, Micropterus salmoides). También, el embalse de Guadalcacín (o de Majaceite), es un punto de pesca de black bass con cierto renombre desde que se capturó el ejemplar de mayor tamaño de España, y se organizan campeonatos de pesca de esta especie. Además, en un radio de $50 \mathrm{~km}$ de los puntos donde se ha encontrado $P$. parva existen 26 embalses, con una superficie entre 0.25 y 3576 hectáreas. En muchos de ellos se practica la pesca de éstas y otras especies invasoras (e.g. Exos lucius), lo que unido al posible uso de $P$. parva como pez pasto, eleva el riesgo de invasión por translocaciones intencionadas hacia otros ríos como los aquí documentados. Una segunda posibilidad implicaría su llegada con el trasvase Guadiaro-Majaceite, que conecta dos cuencas, la Cuenca del Sur y la Cuenca del Guadalquivir (Fig. 1) y enlaza directamente al Río Guadiaro con el embalse del Guadalcacín. Incluso en el caso de que la introducción de $P$. parva no haya llegado desde el embalse del Guadalcacín, este trasvase puede facilitar la dispersión de la especie por la Cuenca del Guadalquivir, lo que supondría un preocupante avance de $P$. parva. La tercera posibilidad vendría dada por la rotura de la presilla de Los Caballeros (también llamada de Montejaque), situada en el tramo alto del Río Guadiaro. En 2010, después de las fuertes precipitaciones caídas entre diciembre de 2009 y febrero de 2010 (2004.8 mm, desviación respecto a la media: $1184 \mathrm{~mm}$ ) se produjo la rotura del di- que de contención. Grupos conservacionistas reportaron el ingreso de black bass y carpa en uno de los arroyos tributarios del Guadiaro (Arroyo de El Cupil), lo que sugiere la posibilidad de que también se produjera la entrada de ejemplares de $P$. parva junto a estas especies. No obstante, creemos que es una opción poco probable dado que el embalse suele secarse con frecuencia.

Respecto a los efectos esperados de esta nueva invasión, junto a la alteración de las redes tróficas y la competencia, debe destacarse el elevado impacto que $S$. destruens provoca en las poblaciones del ciprínido Leucaspius delineatus (Gozlan et al., 2005). L. delineatus y A. hispanica (otro ciprínido ibérico, catalogado como 'En peligro de extinción' para Andalucía (VVAA, 2001), se sitúan dentro del linaje genético de los Alburninae, como ha sido demostrado a través del análisis filogenético de su ADN (Perea et al., 2010). No obstante, los estudios más recientes van aportando evidencias de que tanto $S$. destruens como Anguillicola japonica podrían ser menos selectivos frente a posibles hospedadores de lo que se venía suponiendo (Jackson \& Britton, 2013), lo que advierte de que un amplio número de especies podrían verse negativamente afectadas por estos parásitos además de por la competencia ejercida por P. parva.

Por el momento, dada las características de la especie (rápido crecimiento, carácter prolífico, etc.), el reducido nivel de conocimiento existente sobre sus períodos reproductivos y dinámica poblacional en la Península Ibérica, las dimensiones físicas y ciertos aspectos de la dinámica de los hábitats invadidos (e.g. regímenes de avenida, elevados caudales, etc.), y la reducida eficacia - o imposibilidad de aplicación - de los potenciales métodos de control (despesque, biocidas, desecación, etc.) no parece factible conseguir y mantener una reducción efectiva y duradera de las poblaciones de $P$. parva para reducir los potenciales impactos. Por ello, creemos que el enfoque actual debería centrarse en preservar las poblaciones de especies autóctonas de mayor interés. Para ello, resulta esencial, en una primera fase: a) conocer el área de distribución real de $P$. parva en la región y, muy especialmente identificar la existencia de posibles parásitos exóticos 
asociados a P. parva; b) el seguimiento futuro del estado de las poblaciones de especies de mayor interés para la conservación, que permita diseñar, en los casos necesarios, medidas específicas para cada especie; c) el desarrollo de labores informativas en colaboración con los distintos sectores implicados (Administración y colectivos de pescadores) para concienciar acerca de los impactos que este tipo de especies provocan.

Lo descrito en este trabajo muestra la necesidad de diseñar e implementar medidas efectivas de prevención de la entrada de especies invasoras, que deben pasar, entre otros aspectos, por el refuerzo de la vigilancia en zonas fluviales y por medidas legislativas. En este sentido, la prohibición de la pesca en aquellos puntos donde aparezcan nuevas especies alóctonas se ha considerado una eficaz medida disuasoria frente a la actividad de los 'sembradores' (aquellos que, persiguiendo distintos objetivos, realizan introducciones intencionales de especies invasoras en masas de agua) (Alonso et al., 2000; Bohman et al., 2006; Diéguez-Uribeondo 2006).

\section{AGRADECIMIENTOS}

Este trabajo ha sido financiado por la Consejería de Medio Ambiente y Ordenación del Territorio. Los comentarios a la primera versión del manuscrito realizados por dos revisores anónimos contribuyeron a mejorar el trabajo que presentamos.

\section{REFERENCIAS}

ALONSO, F., C. TEMIÑO \& J. DIÉGUEZ-URIBEONDO. 2000. Status of the white clawed crayfish, Austropotamobius pallipes (Lereboullet, 1858 ) in Spain: distribution and legislation. Bulletin Français de la Pêche et de la Pisciculture, 356: 31-54.

ANDREOU, D., K. D. ARKUSH, J.-F. GUÉGAN, \& R. E. GOZLAN. 2012. Introduced Pathogens and Native Freshwater Biodiversity: A Case Study of Sphaerothecum destruens. PLoS ONE, 7(5): e36998. APARICIO, E., B. PERIS, L. TORRIJOS, J. PRENDA, A. NIEVA \& S. PEREA. 2012. Expansion of the invasive Pseudorasbora parva (Cyprinidae) in the Iberian Peninsula: first record in the Guadiana River basin. Cybium, 36(4): 585-586.

ARKUSH, K. D., S. FRASCA \& R. P. HEDRICK. 1998. Pathology associated with the rosette agent, a systemic protist infecting salmonid fishes. Journal of Aquatic Animal Health, 10: 1-11.

ARKUSH, K. D., L. MENDOZA, M. A. ADKISON \& R. P. HEDRICK. 2003. Observations on the Life Stages of Sphaerothecum destruens n. g., $\mathrm{n}$. sp., a Mesomycetozoean Fish Pathogen Formally Referred to as the Rosette Agent. The Journal of Eukaryotic Microbiology, 50(6): 430-438.

BANARESCU, P. 1964. Pisces-Osteichthyes. Fauna Republicii Populare Romine, volume 13, Editura Academici Republicii Populare Romine, Bucuresti.

BAREA-AZCÓN, J. M., E. BALLESTEROS-DUPERÓN \& D. MORENO. 2008. Libro Rojo de los Invertebrados de Andalucía. 4 Tomos. Consejería de Medio Ambiente, Junta de Andalucía, Sevilla.

BOE-BOLETÍN OFICIAL DEL ESTADO. 2003.

Ley $8 / 2003$, de 28 de octubre, de la Flora y la Fauna Silvestres, BOE 288, Martes 2 de Diciembre de 2003: 42808-42830.

BOE-BOLETÍN OFICIAL DEL ESTADO. 2011. Real Decreto 139/2011, de 4 de febrero, para el desarrollo del Listado de Especies Silvestres en Régimen de Protección Especial y del Catálogo Español de Especies Amenazadas. BOE 46, Miércoles 23 de febrero de 2011, Sec. I: 20912-20951.

BOE-BOLETÍN OFICIAL DEL ESTADO. 2013. Real Decreto 630/2013, de 2 de agosto, por el que se regula el Catálogo español de especies exóticas invasoras. BOE 185, Sábado 3 de agosto de 2013, Sec. I: 56764-56786.

BOHMAN, P., L. EDSMAN \& F. NORMAN. 2006. The effect of large-scale introductions of signal crayfish on the spread of crayfish plague in Sweden 1969-2005. Bulletin Français de la Pêche et de la Pisciculture, 380-381: 1291-1302.

BOJA- BOLETÍN OFICIAL DE LA JUNTA DE ANDALUCÍA. 2012. Decreto 23/2012, de 14 de febrero por el que se regula la conservación y el uso sostenible de la flora y la fauna silvestres y sus hábitats, BOJA 60(2), 27 de Marzo de 2012: 114 163.

BRITTON, J. R. \& R. E. GOZLAN. 2013. How many founders for a biological invasion? Predicting introduction outcomes from propagule pressure. Ecology, 94 (11): 2558-2566. 
CAIOLA, N. \& A. DE SOSTOA. 2002. First record of the Asiatic cyprinid Pseudorasbora parva in the Iberian Peninsula. Journal of Fish Biology, 61: 1058-1060.

DENNY, S. K., A. DENNY \& T. PAUL. 2013. Distribution, prevalence and intensity of Anguillicoloides crassus in the American eel, Anguilla rostrata, in the Bras d'Or Lakes, Nova Scotia. BioInvasions Records, 2(1): 19-26.

DIÉGUEZ-URIBEONDO, J. 2006. The dispersion of the Aphanomyces astaci-carrier Pacifastacus leniusculus by humans represents the main cause of disappearance of the indigenous crayfish Austropotamobius pallipes in Navarra. Bulletin Français de la Pêche et de la Pisciculture, 380-381: 13031312.

DO-DIARIO OFICIAL DE LA COMUNIDAD EUROPEA. 1992. Directiva 92/43/CEE del Consejo, de 21 de mayo de 1992, relativa a la conservación de los hábitats naturales y de la fauna y flora silvestres. Diario Oficial n ${ }^{\circ}$ L 206 de 22/07/1992: 1-59.

DOADRIO, I., S. PEREA, P. GARZÓN-HEYDT \& J. L. GONZÁLEZ. 2011. Ictiofauna continental española. Bases para su seguimiento. DG Medio Natural y Política Forestal. MARM. 616 pp. Madrid.

EIFAC (European Inland Fisheries Advisory Commission) \& ICES (International Council for the Exploration of the Sea). 2008. Report of the 2007 session of the Joint EIFAC/ICES Working Group on Eels Bordeaux, France, 3-7 September 2007. FAO and ICES, Rome, 138 p.

EUROPEAN ENVIRONMENT AGENCY. 2009. Habitats Directive Article 17 Reporting. http://forum. eionet.europa.eu/x_habitat-art17report/library/data sheets/habitats/forests/forests/92b0-riparian_inter mitte/download/1/92B0-Riparian \%20formations $\% 20$ on $\% 20$ intermittent $\% 20$ Med. $\% 20$ courses $\%$ 20Rhodo.pdf [Accessed 1 June 2014]

GALLASTEGI, I., A. RALLO \& M. F. MULCAHY. 2002. A report of Anguillicola crassus from Spain. Bulletin of the European Association of Fish Pathologists, 22: 283-284.

GARCÍA-BERTHOU,E., D. BOIX \& M. CLAVERO. 2007. Non-indigenous animal species naturalized in Iberian inland waters. En: Biological invaders in inland waters: profiles, distribution, and threats. Gherardi, F. (ed.): 123-140. Invading Nature: Springer Series in Invasion Ecology. Springer, Dordrecht, Netherlands.

GOZLAN, R. E., A. C. PINDER \& J. SHELLEY. 2002. Occurrence of the Asiatic cyprinid Pseudo- rasbora parva in England. Journal of Fish Biology, 61: 298-300.

GOZLAN, R. E., S. ST-HILAIRE, S. W. FEIST, P. MARTIN \& M. L. KENT. 2005. Disease threats on European fish. Nature, 435: 1003-1136.

GOZLAN, R. E., E. J. PEELER, M. LONGSHAW, S. ST-HILAIRE \& S. W. FEIST. 2006. Effect of microbial pathogens on the diversity of aquatic populations, notably in Europe. Microbes and Infection, 8: 1358-1364.

GOZLAN, R. E., C.M. WHIPPS, D. ANDREOU \& K. D. ARKUSH. 2009. Identification of a rosettelike agent as Sphaerothecum destruens, a multihost fish pathogen. International Journal of Parasitology, 39: 1055-1058.

GOZLAN, R. E., D. ANDREOU, T. ASAEDA, K. BEYER, R. BOUHADAD, D. BURNARD, N. CAIOLA, P. CAKIC, V. DJIKANOVIC, H. R. ESMAEILI, I. FALKA, D. GOLICHER, A. HARKA, G. JENEY, V. KOVÁČ, J. MUSIL, A. NOCITA, M. POVZ, N. POULET, T. VIRBICKAS, C. WOLTER, A. S. TARKAN, E. TRICARICO, T. TRICHKOVA, H. VERREYCKEN, A. WITKOWSKI, C. G. ZHANG, I. ZWEIMUELLER \& J. R. BRITTON. 2010. Pan-continental invasion of Pseudorasbora parva: towards a better understanding of freshwater fish invasions. Fish and Fisheries, 11(4): 315-340.

HARRELL, L. W, R.A. ELSTON, T. M. SCOTT \& M. T. WILKINSON. 1986. A significant new systemic-disease of net-pen reared Chinook salmon (Oncorhynchus tshawytscha) Brood Stock. Aquaculture, 55: 249-262.

HEDRICK, R. P., C. S. FRIEDMAN \& J. MODIN. 1989. Systemic infection in Atlantic salmon Salmo salar with a Dermocystidium-like species. Diseases of Aquatic Organisms, 7: 171-177.

JACKSON, M. C. \& J. R. BRITTON. 2013. Variation in the trophic overlap of invasive Pseudorasbora parva and sympatric cyprinid fishes. Ecology of Freshwater Fish, 22(4): 654-657.

JURADO-DOÑA, V. 2002. Los bosques de las sierras del Aljibe y del campo de Gibraltar (Cádiz y Málaga). Ecología, transformaciones históricas y gestión forestal. Consejería de Medio Ambiente (Junta de Andalucía), Sevilla.

KNOPF, K. \& M. MAHNKE. 2004. Differences in susceptibility of the European eel (Anguilla anguilla) and the Japanese eel (Anguilla japonica) to the swim-bladder nematode Anguillicola crassus. Parasitology, 129: 491-496. 
LEUNDA, P. M., B. ELVIRA, F. RIBEIRO, R. MIRANDA, J. OSCOZ, M. J. ALVES \& M. J. COLLARES-PEREIRA. 2009. International Standardization of Common Names for Iberian Endemic Freshwater Fishes. Limnetica, 28(2): 189-202.

MAILLO, P. A., M. A. VICH, H. SALVADO, A. MARQUES \& M. P. GRACIA. 2005. Parasites of Anguilla anguilla (L.) from three coastal lagoons of the River Ebro delta (Western Mediterranean). Acta Parasitologica, 50: 156-160.

MENDOZA, L., J. TAYLOR \& L. AJELLO. 2002. The Class Mesomycetozoea: A Heterogeneous Group of Microorganisms at the Animal-Fungal Boundary. Annual Review of Microbiology, 56: 315-344.

PEREA, S., M. BÖHME, P. ZUPANCIC, J. FREYHOF, R. SANDA, M. ÖZULUG, A. ABDOLI \& I. DOADRIO. 2010. Phylogenetic relationships and biogeographical patterns in CircumMediterranean subfamily Leuciscinae (Teleostei, Cyprinidae) inferred from both mitochondrial and nuclear data. BMC Evolutionary Biology, 10: doi: 10.1186/1471-2148-10-265.

PÉREZ-LATORRE, A. V., A. GALÁN DE MERA, P. NAVAS, D. NAVAS, Y. GIL \& B. CABEZUDO. 1999. Datos sobre la Flora y Vegetación del Parque Natural de los Alcornocales (Cádiz-Málaga, España). Acta Botanica Malacitana, 24: 133-184.

POLLUX, B. J. A. \& A. KOROSI. 2006. On the occurrence of the Asiatic cyprinid Pseudorasbora par$v a$ in the Netherlands. Journal of Fish Biology, 69: 1575-1580.

POU-ROVIRA, Q., M. CLAVERO \& L. ZAMORA. 2007. Els peixos de les Gavarres i entorns (Fishes of the Gavarres mountains and its surroundings). Biblioteca Lluís Esteva, vol 5. Consorci de les Gavarres, Girona.

PRENDA, J., F. BLANCO \& M. CLAVERO. 2003. Los peces continentales de la provincia de Cádiz. Revista de la Sociedad Gaditana de Historia $\mathrm{Na}$ tural, 3: 173-183.

RUIZ-GARCÍA, A., A. F. HERRERA-GRAO \& M. FERRERAS-ROMERO. 2006. Distribution of Trichoptera Communities in the Hozgarganta Catchment (Los Alcornocales Natural Park, SW Spain). International Review of Hydrobiology, 91(1): 71-85.

SIMON, A., R. BRITTON, R. GOZLAN, C. VAN OOSTERHOUT, F.A.M. VOLCKAERT \& B. HÄNFLING. 2011. Invasive Cyprinid Fish in Europe Originate from the Single Introduction of an Admixed Source Population Followed by a Complex Pattern of Spread. PLoS ONE, 6(6): e18560

SPIKMANS, F., T. VAN TONGEREN, T. A. VAN ALEN, G. VAN DER VELDE \& H. J. M. OP DEN CAMP. 2013. High prevalence of the parasite Sphaerothecum destruens in the invasive topmouth gudgeon Pseudorasbora parva in the Netherlands, a potential threat to native freshwater fish. Aquatic Invasions, 8(3): 355-360.

VERDÚ, J. R. \& E. GALANTE (eds.). 2009. Atlas de los Invertebrados Amenazados de España (Especies En Peligro Crítico y En Peligro). Dirección General para la Biodiversidad, Ministerio de Medio Ambiente, Madrid, Spain.

VVAA. 2001. Libro Rojo de los Vertebrados Amenazados de Andalucía. Consejería de Medio Ambiente, Junta de Andalucía, Sevilla, Spain. 